\title{
Unilateral Four-Headed Pectoralis Muscle Major
}

\author{
Rasheed Bani Hammad*, Adel Mohamed ${ }^{\dagger}$
}

\begin{abstract}
A unilateral four-headed pectoralis major muscle was observed on the left side of an 83 year-old Caucasian male cadaver. The accessory tendon arises from the lateral aspect of the pectoralis major muscle, travels along with the tendon of the long head of the biceps brachii and blends into the capsule of the shoulder joint. This anomaly is rare. A complete or partial absence of pectoralis major muscle is normally reported. Additionally, supernumerary heads of muscles other than pectoralis major have been documented. However, the existence of an accessory tendon to the pectoralis major muscle is unique. The implications of such a finding are discussed.
\end{abstract}

KEY WORDS: Accessory tendon, biceps brachii, pectoralis major, Poland's Syndrome.

\section{INTRODUCTION}

The pectoralis major muscle in humans is a large fan shaped muscle situated at the anterior upper part of the chest. It is complex in that it has two separate muscular bellies and a dual nerve supply. The clavicular portion of the pectoralis major muscle arises from the medial one half to two thirds of the clavicle, and passes downward and laterally to its humeral insertion. The sternocostal portion arises from the anterior surface of the sternum's second to the sixth costal cartilages, and from the aponeurosis of the external oblique muscle. From these two origins, the fibers converge toward their insertions: those arising from the clavicle pass obliquely downward and laterally, and are usually separated from the rest by a slight interval; those from the lower part of the sternum, and the cartilages of the lower true ribs, run upward and laterally, while the middle fibers pass horizontally. They all end in a flat tendon, which is

\footnotetext{
*To whom correspondence should be addressed:

CAMECO MS Neuroscience Research Center (CMSNRC), Saskatoon City Hospital, 701 Queen Street. Rm. 5800, Saskatoon, (SK), S7K 0M7; email: rab219@ mail.usask.ca

$\dagger$ Department of Anatomy and Cell Biology, University of Saskatchewan College of Medicine, 107 Wiggins Road, Saskatoon, Saskatchewan, SK, S7N 5E5
}

inserted into the lateral lip of the intertubercular sulcus of the humerus (1). The pectoralis major muscle is innervated by lateral (C5 and C6) and medial pectoral (C7, C8, and T1) nerves. The pectoralis major muscle adducts and medially rotates the humerus, and draws the scapula anteriorly and inferiorly. The clavicular head acts to flex the humerus, and the sternocostal head extends it from flexed position. It also acts as an accessory muscle for inspiration. The muscle is supplied by the pectoral branch of the thoracoacromial trunk artery, and by branches of the lateral thoracic artery. It is also a dynamic stabilizer of the shoulder joint; therefore, it is important in upper limb mobility, and the presence of supernumerary large heads may provide additional strength to the biceps brachii as described by Swieter and Carmichael (2).

\section{THE CASE}

Here we report a unique case of unilateral four-head pectoralis major muscle in an 83 year-old cadaver (Figure 1), that was otherwise anatomically and functionally unremarkable. We observed the accessory tendon of the pectoralis major muscle on the left side of this male cadaver. The accessory tendon arises from the lateral aspect of the pectoralis major muscle. The accessory tendon has approximately the same 


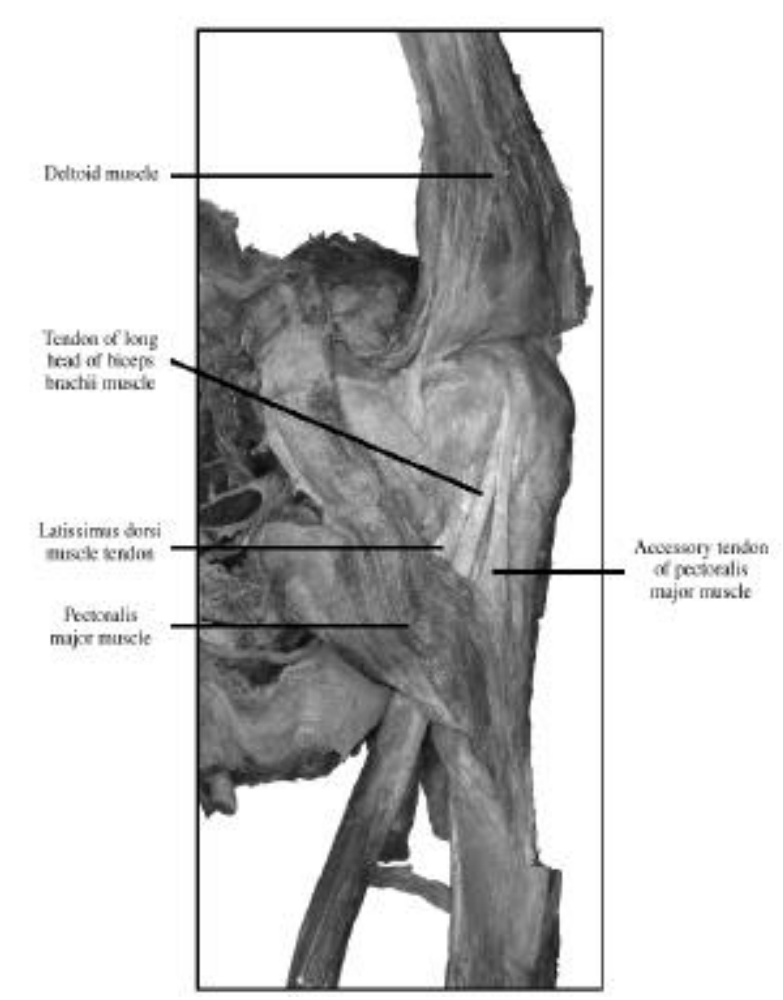

Figure 1. Frontal view of the left shoulder showing the anatomy of the accessory tendon. The deltoid muscle has been reflected.

dimensions and position as the tendon of the long head of the biceps brachii muscle. In fact, the accessory tendon travels alongside the tendon of the long head of the biceps brachii muscle (Figure 2.). This accessory tendon is located directly lateral to the tendon of the long head of the biceps brachii muscle. The position of this accessory tendon obscures the view of the biceps tendon when the pectoralis major is not retracted. Proximally, the accessory tendon blends into the fibers of the capsule of the shoulder joint. Distally, the accessory tendon attaches to and blends in with the superior fibers of the pectoralis major tendon, which inserts into the lateral lip of the intertubercular sulcus of the humerus. No abnormality was observed in the nerve and blood supply either to the pectoralis major muscle or to the accessory tendon.

\section{DISCUSSION}

The literature documents numerous variations associated with the pectorlis major muscle. The complete absence of the pectoralis major is rare $(1,3)$. Gray described some variations associated with the pectoralis muscle such as greater or lesser extent of attachment to the ribs and sternum, varying size of the abdominal part or its absence, greater or lesser extent of separation of sternocostal and clavicular parts, fusion of clavicular part with deltoid, and decussation in front of the sternum. Deficiency or absence of the sternocostal part is not uncommon. Absence of the clavicular part is less frequent (1). Occasionally, parts of the pectoralis major muscle may be absent. The sternocostal origin is the most commonly missing part, lack of which causes weakness in the adduction and medial rotation of the shoulder joint (4). In addition to the variations within the pectoralis muscle, Gray also described the rare presence of additional muscles such as costocoracoideus, chondro-epitrochlearis, and sternalis, all of which are closely associated with the pectoralis major (1).

Although most of the variations mentioned above are associated with the surface anatomy of the bellies of the pectoralis muscle, the presence of an accessory tendon in the pectoralis muscle has never been described.

The presence of an accessory tendon to the pectoralis major muscle might stabilize the muscle origins (2). However, premortem clinical history was unremarkable regarding impairment of shoulder movements. This indicates that the presence of such an anomaly may not impede normal movements of the shoulder.

Most of the reported pectoralis major abnormalities focused on its complete or partial absence due to congenital reasons (5-10), or as a result of diseases such as leukemia (11) and Poland's syndrome, where absence of the costosternal portion of the pectoralis major has been reported $(12,13)$. Poland's syndrome is a group of unilateral congenital abnormalities of the chest wall with or without involvement of the arm on the same side. The right side is affected twice as often as the left side and there is a male preponderance. Pectoralis major abnormalities included bilateral (14) or unilateral absence of the pectoralis major (15). However, regarding the presence of accessory tendons of the

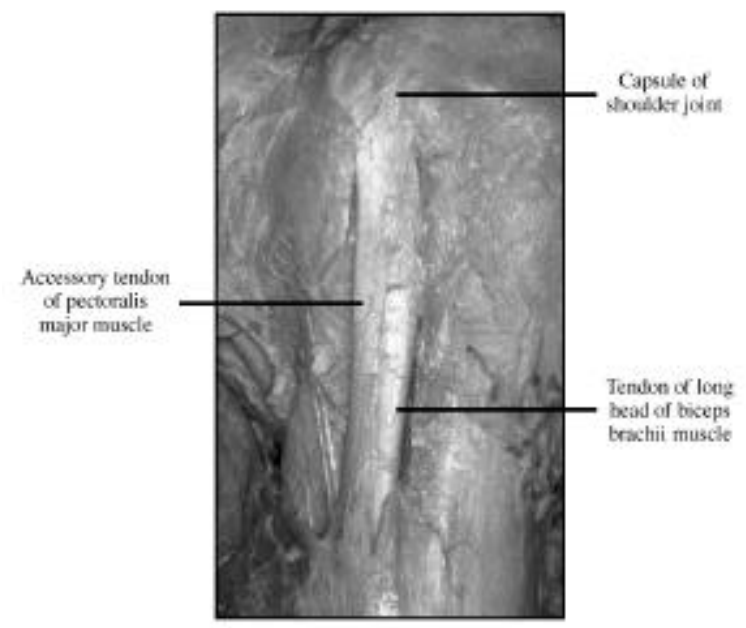

Figure 2. In this view the accessory tendon of the pectoralis muscle clearly runs alongside the tendon of the long head of the biceps brachii muscle, and both insert in the shoulder joint capsule area 
pectoralis muscle, studies reported accessory fibrous bands arising from the lateral border of the pectoralis that have intimate contact with the median nerve (16).

We believe that the variation reported in this region is important both functionally and clinically. This finding is of clinical importance in transplant surgery, giving additional options for insertion of tendons in the shoulder joint area. Additionally, the fact that we found this unique case in a male cadaver raises the question whether it is sex-specific, and encourages a more thorough search to find out if a similar pattern of variation exists in females. Should this pattern be present in females, it would be of clinical importance in cases of breast or transplant surgeries.

\section{REFERENCES}

1. Gray H. In: Gray's Anatomy. New York Williams-WarwickDyson-Bannister, 1989.

2. Swieter, M.G. and Carmichael. Bilateral three-headed biceps brachii muscles. Anatomischer Anzeiger. 148:346-349; 1980.

3. Beals RK and Crawford S. Congenital absence of the pectoral muscle. Clinical orthopaedics. 1976; 119:166.

4. Snell RS. In: Clinical Anatomy for Medical Students. Baltimore, MD. Boston Little, Brown and Company, 1995.

5. Pierach CA. [Missing major pectoral muscle] (German). Fortschritte auf dem Gebiete der Rontgenstrahlen und der
Nuklearmedizin. 1973; 118(2): 225-6.

6. Samilson R L. Congenital and developmental anomalies of the shoulder girdle. Orthopedic Clinics of North America. 1980; 11(2): 219-231.

7. Segall HD. Congenital absence of the pectoralis major muscle. Medical Radiography and Photography. 1972; 48(1): 23.

8. Almstead, S. Congenital defects of the pectoral muscles. Lancet. 1933; 1:1179.

9. Christopher, F. Congenital absence of the pectoral muscles. The Journal of Bone and Joint Surgery. 1928; 10:350-351.

10. Samuel. E. Congenital absence of the pectoralis major. British Journal of Radiology. 1945; 18:20-21.

11. Armendares S. Letter: Absence of pectoralis major muscle in two sisters associated with leukemia in one of them. The Journal of Pediatrics. 1974; 85(3): 436-7.

12. Frias JL, Felman AH. Absence of the pectoralis major, with ipsilateral aplasia of the radius, thumb, hemidiaphragm and lung: an extreme expression of poland anomaly? Birth Defects Original Article Series. 1974; 10(5): 55-9.

13. Urschel HC Jr. Polands syndrome. Chest Surgery Clinics of North America. 2000; 10(2): 393-403.

14. Mosconi T, Kamath S. Bilateral asymmetric deficiency of the pectoralis major muscle. Clinical Anatomy. 2003; 16(4):346-9.

15. Debeer P, Brys P, De Smet L, Fryns JP. Unilateral absence of the trapezius and pectoralis major muscle: a variant of Poland syndrome. Genetic Counseling. 2002; 13(4):449-53.

16. Yazar F, Kirici Y, Ozan H. Accessory insertions of pectoralis major muscle to the brachial fascia: a case report. Kaibogaku Zasshi Journal of Anatomy. 1998; 73(6): 637-9.

\footnotetext{
Rasheed Bani Hammad is a third year PhD. Student in the Department of Anatomy and Cell Biology, University of Saskatchewan College of Medicine. He is funded by the United Arab Emirates University Scholarship Program. $\mathrm{He}$ is currently working in understanding the cell biology of sensory neurons to peripheral nerve injury. He obtained his Bachelor's of Science Degree from Villanova University, PA., USA. He earned his Master's Degree in Cardiogenesis from Temple University, PA., USA.

Adel Mohamed, MD, qualified by American Board of Pathology in Anatomical and Clinical Pathology, Cytopathology fellowship, Anatomy fellowship, is currently an associate professor of Anatomy and Cell Biology and Associate member of Pathology and Lab Medicine and Medical and Dental Gross Anatomy Coordinator, College of Medicine, University of Saskatchewan, Saskatoon.
} 